\title{
Maintenance Treatment for the Modulation of Liver Fibrosis
}

\author{
Evaldo Stanislau Affonso de Araújo and Antonio Alci Barone \\ Laboratory of Hepatitis, LIM 47 DMIP-HC-FMUSP; São Paulo, SP, Brazil
}

In many cases, the evolution of chronic infection with the hepatitis $\mathrm{C}$ virus (HCV) is favorable. However, progression to liver fibrosis is a common phenomenon and can lead to liver cirrhosis. Phenomena associated with liver fibrosis have been previously reviewed and are more related to the host than to viral factors [1]. In brief, the onset of fibrosis is caused by the activation of stellate cells, which acquire the shape of myofibroblasts and become the source of collagen deposition, as well as of protein matrix formation. Activated stellate cells rapidly undergo apoptosis, after which the collagen matrix is degraded and removed by the activity of metalloproteinases. There is a complex balance between the procollagen and antifibrotic factors, although the mechanisms of fibrosis regression are not fully understood [2]. However, it appears that the tissue inhibitor of metalloproteinase-1 [3] and stellate cell apoptosis [4] are crucial to maintaining this balance. The mechanism through which HCV triggers fibrosis is little understood. Apparently, hepatocyte infection triggers a state of oxidative stress and induces inflammatory cell recruitment. These phenomena lead to the activation of stellate cells and collagen deposition. In addition, HCV proteins directly activate stellate cells [4].

Liver cirrhosis and the preceding transition stage are marked phenomena in the clinical evolution of patients and have been associated with morbidity/mortality due to chronic hepatitis C. A study involving a cohort of patients chronically infected with HCV, monitored from 1991 onward, with a mean infection period of 22 years, provided evidence that, five years after the diagnosis of cirrhosis (Ishak score $\geq 4$ ), the survival rate was $80 \%$ [5]. This rate decreased to $19 \%$ after the first hepatic decompensation [5]. In the multivariate analysis, treatment with the combination of conventional or pegylated interferon alpha with ribavirin was found to be favorably associated with survival. In other words, the treatment was associated with the most important outcome: survival! In addition, subjects presenting a virological response, whether sustained or not, presented better evolution when compared to nonresponders. This fact suggests that even transitory negative viremia levels are a favorable phenomenon and may imply lower structural alterations and activity in the liver [5]. One relevant aspect of this study was that the interruption of alcohol consumption from the moment of diagnosis on prevented the previous ingestion from having an unfavorable influence on the disease progression. This fact reinforces the importance of alcohol abstinence for patients with hepatitis C. Poynard et al. [6] retrospectively analyzed data from 3,010 patients treated with either conventional or pegylated

The Brazilian Journal of Infectious Diseases 2007;11 (5) Suppl. 1:52-55. (C) 2007 by The Brazilian Journal of Infectious Diseases and Contexto Publishing. All rights reserved. interferon alpha, using various therapeutic regimens . The authors concluded that the treatment with pegylated interferon and ribavirin resulted in significant improvement in histology, inflammatory activity and structural alterations (fibrosis). There was improvement in the 'fibrosis progression rate', which is a valid concept, although reproducibility was jeopardized by the risks of sample variation. The least worsening of fibrosis was found in the optimized group receiving pegylated interferon and ribavirin (8\%), and greatest degree of such worsening was found in the group receiving interferon for 24 weeks (23\%) [6]. In general, fibrosis stabilized or improved even in those patients not achieving a sustained virological response (SVR). However, sample variations, a high percentage of patients with mild initial fibrosis (over 70\% classified as F1), lack of paired biopsy results from all participants, lack of a control group, and principally, a relatively short follow-up period (20 months between biopsies, on average) were limitations of this analysis. Nevertheless, Poynard et al. [6] addressed the concept of cirrhosis 'reversion', or as the authors designated it, the 'reversible cirrhosis stage'. This group was composed of young patients, whose structural staging changed, regressing from F4. This phenomenon occurred in 75 (49\%) of the 153 cirrhotic patients [6]. The authors postulated that this stage of fibrosis would still be 'easily' reversed. Some of the factors that were found to be associated with the regression of fibrosis after treatment are, obviously, the initial degree of fibrosis, minimal baseline activity, achieving an SVR, being less than 40 years of age, initial viral load lower than 3.5 million copies/mL, and (a new concept at that time) body mass index (BMI) $<27 \mathrm{~kg} / \mathrm{m}^{2}$ [6]. In another relevant meta-analysis, Cammà et al $[7,8]$. evaluated three randomized clinical studies comprising 1441 patients, paired biopsies being available for 1013 (70.3\%). Similarly to Poynard et al. [6], these authors demonstrated improvement in hepatic fibrosis in the patients treated with pegylated interferon alpha-2a, when compared to those treated with conventional interferon, who achieved an SVR or even experienced recurrence. However, there was no improvement among nonresponders. The authors also found that a BMI higher than $30 \mathrm{~kg} / \mathrm{m}^{2}$ was associated with the worsening of hepatic fibrosis. However, in a more representative sample 447 (44\%) of 1013 cirrhotic patients - no 'regression of cirrhosis' was found. Only 33\% of the cirrhotic patients presented improvement in fibrosis. However, the observation period between biopsies was also short. Finally, high alanine aminotransferase level was another factor that was associated with histological improvement. More recently, Di Marco et al. [9] prospectively evaluated cirrhotic patients with portal hypertension and no previous decompensation who received pegylated interferon alpha-2b $(1.0 \mu \mathrm{g} / \mathrm{kg} /$ week $)$ with or without 
ribavirin (0.8 g/day). Patients infected with genotype 2 or 3 showed rapid response, with negative viral RNA results by week 4 of treatment, and achieved an SVR. However, only 10 of the patients infected with genotype 1 or 4 achieved an SVR. Nevertheless, for this subgroup of patients, the predictability of early response by week 12 , but not by week 4, was also valid, and high baseline viral load was a negative predictive marker for SVR. The occurrence of cytopenias was high; however, stimulating factors were not used with these patients. If stimulating factors had been available, the SVR rate associated with treatment maintenance would have certainly been better. Treatment compliance is even more critical for this group of patients and was associated with SVR [9]. Finally, it was clear that SVR is associated with better evolution. Only 6\% of the patients achieving an SVR deteriorated, compared with 38\% of the nonresponders [9]. Bruno et al. [10] conducted a retrospective multicenter study comprising 920 patients with compensated cirrhosis who were treated with conventional interferon alpha (from 3 to 6 million IU/3 times a week) for a year. Similarly to the prospective study conducted by Di Marco et al. [9], the authors demonstrated benefits for those who achieved an SVR, with a reduction in the risk of decompensation, occurrence of hepatocellular carcinoma (HCC) and death. They also found that a platelet count of $109,000 / \mathrm{mL}$ is an independent predictor of decompensation of the liver disease [10]. This factor is extremely relevant for clinical practice and could be used as a cut-off point in order to define the use of more aggressive measures, or even those that still need to be validated. Specifically regarding HCC, Cammà et al. [7,8] in 2001, pointed out that the benefit of the reduction in the incidence of HCC, albeit modest, is also more relevant among those who achieve an SVR after being treated with interferon alpha. Other authors have also reproduced data regarding the effective and tolerable treatment [11], fewer complications, reduction or negative incidence of HCC among cirrhotic patients monoinfected with HCV who achieved an SVR after the treatment with interferon alpha [12-14], and even histological response of patients co-infected with HIV [12]. Finally, even patients with severe cirrhosis under individualized, ascending-dose regimens benefit from treatment and can achieve an SVR, albeit a modest one [15-17]. Despite the innumerable benefits of the treatment with interferon alpha, we must emphasize that surveillance regarding the incidence of HCC is highly recommended, even in cirrhotic patients who have achieved an SVR [10].

There is clear evidence of improvement in fibrosis (a quantitative decrease, as well as functional gains such as lower portal hypertension) [3], greater survival, potential decrease in the incidence of HCC and hepatic complications, and even 'cirrhosis reversion' [6], even for nonresponders [6] or for those who presented recurrence [5-8]. Therefore, we should ask why we do not treat even those patients with more severe cirrhosis [15-17], and why we do not use the treatment with interferon alpha for objectives other than the virological.
In order to answer these questions, it is initially important that recent knowledge on viral kinetics and the concepts of treatment individualization - dose and duration -be considered in the therapeutic decision-making, and that the same concepts are not clearly validated for cirrhotic patients. Therefore, if our goal is to achieve an SVR, extending the treatment of nonresponders to at least 24 weeks of 'ideal' treatment is considered 'futile' [1]. However, is this true from a histological point of view, or from the perspective of the need to modulate the natural history of the disease? In order to answer these questions and in view of the previously described evidence, physicians began to consider the use of maintenance treatment with interferon alpha. However, for a conclusive analysis, a prospective evaluation would be ideal, since differences in methodologies would greatly affect the quality of the results [17]. Three principal studies (Table 1) have addressed this issue: the Hepatitis C Antiviral LongTerm Treatment against Cirrhosis (HALT-C) trial, conducted by the NIH; the Evaluation of Peg-Intron in Control of Hepatitis C Cirrhosis (EPIC)3 trial; and the Colchicine versus Peg-Interferon Long-Term (COPILOT) trial. Other, smaller, studies, such as the PROFIC-C trial, have also addressed this issue [18].

The COPILOT study evaluates patients with fibrosis classified as greater than Ishak 3 and previous nonresponders to interferon/ribavirin or pegylated interferon/ribavirin, comparing, in two branches, colchicine to pegylated interferon alpha-2b at $0.5 \mu \mathrm{g} / \mathrm{kg} /$ week. Preliminary analyses after a twoyear follow-up period revealed that the group using interferon presented significantly fewer hepatic complications, especially portal hypertension and upper gastrointestinal tract bleeding [6]. Alterations in HCV quantification were minimal. The EPIC3 trial has yet to produce preliminary results. However, the HALT-C study has provided a consistent amount of information. Nevertheless, data regarding the main objective of the study, fibrosis modulation, are still unavailable but should be presented at the upcoming congress of the American Association for the Study of Liver Diseases (AASLD; Afdhal, personal communication). Using the available results from HALT-C, Everson et al. [15], in 2006, emphasized the need to 'optimize' the treatment of cirrhotic patients, for whom the SVR rate was lower, regardless of platelet counts or the need to reduce interferon doses - or even the influence of previous treatment response. Therefore, cirrhosis is a determining factor of a lower SVR rate. Among the therapy ‘optimization’ measures that are currently available, should we consider treatment prior to the establishment of cirrhosis? What would be the criteria? We still cannot answer that, but if we consider some preliminary results of new therapies, we can predict that interferon alpha will still be the backbone of hepatitis treatment for many years. Therefore, we should certainly attempt to gain a better understanding of the potential of these treatments and use them wisely.

Among the minor studies, Erhardt et al. preliminarily showed that the maintenance of a $0.35-1.0 \mu \mathrm{g} / \mathrm{kg} /$ week dose 
Table 1. Maintenance studies with pegylated interferon

\begin{tabular}{|c|c|c|c|}
\hline Study & HALT-C & COPILOT & EPIC3 \\
\hline \multirow[t]{2}{*}{ Disease stage } & Ishak 4-6 & Ishak 3-6 & METAVIR 2-4 \\
\hline & $\mathrm{CTP} \leq 6$ & $\mathrm{CTP} \leq 7$ & $\mathrm{CTP} \leq 6$ \\
\hline Patients (n) & 1400 & 800 & 1700 (700 cirrhotic) \\
\hline Treatment arms & Placebo, IFN- $\alpha$, Peg-IFN- $\alpha-2 \mathrm{a}(90 \mu \mathrm{g})$ & Peg-IFN- $\alpha-2 b(0.5 \mu g)$ & \\
\hline Colchicine (0.6 mg bid) & Peg-IFN á-2b (0.5 ㅆg) & & \\
\hline Duration & 3.5 years & 4 years & 3-5 years \\
\hline
\end{tabular}

HALT-C=Hepatitis C Antiviral Long-Term Treatment against Cirrhosis (trial); COPILOT= Colchicine versus Peg-Interferon Long-Term (trial); EPIC3=Evaluation of Peg-Intron in Control of Hepatitis C Cirrhosis (trial) 3; CTP=Child-Turcotte-Pugh score; IFN=interferon; Peg=pegylated.

of pegylated interferon alpha-2b significantly reduced, after 48 weeks, the incidence of HCC and complications due to cirrhosis when compared to the control group. In a similar study, Kaiser et al. [19] demonstrated that, in the intervention group, the fibrosis score dropped from 3.58 to 2.59 after 18 months of treatment, and to 2.36 by six months after the end of treatment. In the control group, the fibrosis score increased from 3.88 to 4.07 and to 4.79 by the same time points. Therefore, monotherapy with lower doses of pegylated interferon alpha$2 \mathrm{~b}$ effectively reduced and modulated hepatic fibrosis. The mechanism for the improvement induced by interferon is unknown and is certainly multifactorial. The elimination of the triggering agent is undoubtedly crucial, but, since even patients who suffer recurrence get better, it is possible that interferon alpha has an intrinsic antifibrotic effect, as well as inhibiting the activation of stellate cells [4]. Other possible approaches that are positively associated with the improvement of hepatic fibrosis in nonresponders - or as a coadjuvant - are the use of renin-angiotensin inhibitors (inhibiting the activation of stellate cells) and the control of metabolic syndrome [4]. Other substances that are potentially active against hepatic fibrosis are interleukin-10 and the natural herb known as Sho-saiko-to [4]. The maintenance treatment with ribavirin is clearly a discarded alternative since there are no positive effects [20]. Finally, the use of controlled phlebotomies may be an alternative in selected cases. Excessive iron in the hepatocytes, increased by the effect of $\mathrm{HCV}$, has been associated with greater tissue damage [20]. The field of antifibrogenesis is in full development and has been recently reviewed in an AASLD symposium [3].

Considering all the reviewed aspects, we should finally evaluate who would benefit from the maintenance treatment. These would be the patients with extensive fibrosis (F3/F4) or cirrhosis that does not respond to the optimal standard treatment. In view of the risk of disease progression and the onset of HCC, as well as the body of evidence available, simply monitoring the progression of the disease is not an acceptable approach, neither for the patient nor for the physician [20]. Therefore, what is the ideal dose and how long should we maintain the treatment with interferon? The period has yet to be defined, and it may be indefinite (or until the appearance of a definitely efficacious, safe therapy). Biopsy monitoring every two years in association with HCC and screening for esophageal varices are acceptable standards, although normalization of alanine aminotransferase levels and HCV reduction will rarely be seen [20]. The advent of noninvasive methods of monitoring fibrosis [21,22] has transformed maintenance into an even more alternative strategy. Regarding the adopted dose, in ongoing and published studies, pegylated interferon alpha has been used in smaller-thanhabitual doses, once a week. Tolerance and safety have proven adequate. Doses from one-third to one-half of the standard size seem to be satisfactory for hepatic fibrosis modulation. However, definitive results, which are still unavailable, could alter this perception.

In summary:

1. Hepatic fibrosis is a potentially reversible phenomenon, which can even provide functional benefits.

2. Even for cirrhotic patients, treatment with interferon alpha is possible, efficient and safe, although it is less efficacious if we consider the SVR.

3. Treatment with pegylated interferon alpha, even when it is ineffective, is associated with greater survival, fewer complications and lower incidence of HCC.

4. Interferon alpha has a modulatory effect on hepatic fibrosis.

5. Maintenance treatment with reduced doses of pegylated interferon alpha proved to be effective in modulating hepatic fibrosis as well as in altering the natural history of the disease.

6. Antifibrogenesis is a field that is still in development, and, in addition to interferon alpha, other measures can be adopted, such as control of metabolic syndrome and the use of renin-angiotensin inhibitors.

Considering the reviewed aspects, together with the facts that the number of antiviral therapies currently in development is smaller than expected, and that none of those are yet clinically available, as well as the fact that they will still need to be combined with interferon alpha, I believe that the optimization of interferon alpha will continue to be crucial. A pharmaco-economic analysis must be certainly considered as a supporting tool in the collective decision-making. However, considering the physician-patient relationship, some reflections are pertinent and should be debated by the Hepatitis Committee of the Brazilian Society of Infectology. 
Therefore, we propose the following:

\section{Individualization}

The dose and duration of treatment with the combination of pegylated interferon and ribavirin should be defined based on patient body weight and on the early response during therapy for patients with minimal lesions (F1) who nevertheless are at risk for progression: moderate peri-portal activity, from 40 to 60 years of age and comorbidities (nonalcoholic steatohepatitis, HIV infection or metabolic syndrome).

\section{Maintenance Treatment}

Considering maintenance treatment with pegylated interferon alpha for patients with structural lesions greater than F3 or portal hypertension signs (esophageal varices, enlarged spleen, dilation of vessels) or platelet counts lower than $110,000 / \mathrm{mm}^{3}$, classified as Child-Pugh class A or B, no evidence of severe or potentially uncontrollable decompensation, with no HCC, no recurrence, partial responders or nonresponders to pegylated interferon alpha in association with ribavirin (or only to interferon when there are contraindications regarding ribavirin) whose compliance can be confirmed for at least 12 weeks. This treatment should also be considered for patients who present contraindications regarding the use of full doses. Child-Pugh class $C$ patients could be treated in specialized centers when on a transplant waiting list.

Proposed regimen*: subcutaneous pegylated interferon alpha-2b at 0.5-1.0 $\mu \mathrm{g} / \mathrm{kg} /$ week; subcutaneous pegylated interferon alpha-2a at $90 \mu \mathrm{g} /$ week.

Duration*: at least 24 months, indefinite, or until the appearance of a definitely efficacious, safe antiviral therapy.

Follow-up evaluation: Monitoring the onset of complications (HCC, digestive tract hemorrhage, encephalopathy, etc.)

*Dose schedule and duration were suggested based on preliminary data and should be reassessed depending on the results of ongoing studies.

\section{References}

1. Hoofnagle J.H., Seef L.B. Peginterferon and ribavirin for chronic hepatitis C. New England Journal of Medicine 2006;355(23):2444-51.

2. Arthur M.J.P.Reversibility of liver fibrosis and cirrhosis following treatment for hepatitis C.Gastroenterology 2002;122(5):15258.

3. Friedman S.L., Rockey D.C., Bissel M. Hepatic fibrosis 2006: report of the third AASLD single topic conference. Hepatology 2007;45:242-9.
4. Bataller R., Brenner D.A. Liver fibrosis. The Journal of Clinical Investigation 2005;115:209-18.

5. Lawson A., Hagan S., Rye K., et al. The natural history of hepatitis C with severe hepatic fibrosis. Journal of Hepatology 2007; 47:37-45.

6. Poynard T., McHutchinson J., Manns M., et al. Impact of pegylated interferon alfa-2b and ribavirin on liver fibrosis in patients with chronic hepatitis C. Gastroenterology 2002;122:1303-13.

7. Cammà C., Di Bona D., Schepis F., et al. Effect of peginterferon alfa-2a on liver histology in chronic hepatitis C: a meta-analysis of individual patient data. Hepatology 2004;39:333-42.

8. Cammà C., Giunta M., Andreone P., Craxì A. Interferon and prevention of hepatocellular carcinoma in viral cirrhosis: an evidence-based approach. Journal of Hepatology 2001;34:593602.

9. Di Marco V., Almasio P.L., Ferraro D., et al. Peg-Interferon alone or combined with ribavirin in HCV cirrhosis with portal hypertension: a randomized controlled trial. Journal of Hepatology 2007 (in press).

10. Bruno S., Stroffolini T., Colombo M., et al. Sustained Virological Response to interferon-á is associated with improved outcome in HCV-related cirrhosis: a retrospective study. Hepatology 2007;45:579-87.

11. Helbling B., Jochum W., Stamenic I., et al. HCV-Related advanced fibrosis/cirrhosis: randomization controlled trial of pegylated interferon á-2a and ribavirin. Journal of Viral Hepatitis 2006; $13: 762-9$.

12. Sarmento-Castro R., Horta A., Vasconcelos O., et al. Impacto f peginterferon alpha-2b and ribavirin treatment on liver tissue in patients with HCV or HCV-HIV co-infection. Journal of Infection 2007;54:609-16.

13. Veldt B.J., Saracco G., Boyer N., et al. Long term clinical outcome of chronic hepatitis $C$ patients with sustained virological response to interferon monotherapy. Gut 2004;53:1504-8.

14. Coverdale S.A., Khan M.H., Byth K., et al. Effects of interferon treatment response on liver complications of chronic hepatitis C: 9-year follow-up study. American Journal of Gastroenterology 2004.

15. Everson T.G., Hoefs J.C., Seef L.B., et al. Impact of disease severity on outcome of antiviral therapy for chronic hepatitis C: lessons from the HALT-C Trial. Hepatology 2006;44:1675-84.

16. Everson T.G., Trotter J., Forman L., et al. Treatment of advanced hepatitis $\mathrm{C}$ with low accelerating dosage regimen of antiviral therapy. Hepatology 2005;42:255-62.

17. Everson G.T. Maintenance Interferon for chronic hepatitis C: more issues than answers? Hepatology 2000;32:436-8.

18. Schuppan D., Krebs A., Bauer M., Hahn E.G. Hepatitis C and liver fibrosis. Cell Death and Differentiation 2003;10:S59-S67.

19. Kaiser S., Hass H., Luize B., et al. Long term, low dose treatment with pegylated interferon alfa $2 \mathrm{~b}$ leads to a significant reduction in fibrosis and inflammatory score in chronic hepatitis $\mathrm{C}$ nonresponder patients with fibrosis or cirrhosis.41 ${ }^{\text {st }}$ EASL, 2006.

20. Kelleher T.B., Afdhal N.H. Maintenance therapy for chronic hepatitis C. Current Gastroenterology Reports 2005;7:50-3.

21. Afdhal N.H., Kowdley K.V., Llovet J.M. CCO Independent Conference Coverage of the 2007 Annual Meeting of EASL. Interim analisys: peginterferon alfa-2b maintenance therapy may reduce incidence of HCV-related HCC, Cirrhosis complications, 2007.

22. Afdhal N.H., Nunes D. Evaluation of liver fibrosis: a concise review. American Journal of Gastroenterology 2004;1160-70. 